\title{
ATUALIZAÇÃO TÉCNOLOGICA DA DESSULFURAÇÃO PRIMARIA E SUBSTITUIÇÃO DE DESSULFURANTE A BASE DE CAC 2 POR PURESTEEL*
}

\author{
Paulo César Pontelo 1 \\ Marcos Santos ${ }^{2}$ \\ Alisson Alves de Oliveira ${ }^{3}$ \\ Alan Jardim Nascimento ${ }^{4}$ \\ Uander Henrique de Freitas ${ }^{5}$
}

\section{Resumo}

Buscando atender seus clientes com soluções inovadoras no campo do tratamento de metais líquidos, a Sidertec efetuou a atualização tecnológica do sistema de dessulfuração primária de uma grande usina integrada brasileira trazendo o seu sistema antes obsoleto para o estado da arte em dessulfuração e ainda substituindo o dessulfurante a base de $\mathrm{CaC}_{2}$ anteriormente utilizado por PureSteel®; tendo como benefícios a otimização do processo, redução de custo de $61 \%$ e melhora na condição de segurança operacional pela retirada de produto perigoso do processo da usina. Esta implementação utilizou o Know How Sidertec e se baseou na metodologia de estudo do problema, aprofundamento de engenharia das possíveis soluções e realização do revamping proposto.

Palavras-chave: Dessulfuração; Carro torpedo; Tecnologia.

\section{PRIMARY DESSULPHURIZATION STATION UPDATE AND CAC2 BASE DESSULPHURIZER REPLACEMENTE FOR PURESTEEL}

\begin{abstract}
Seeking to meet its clients with innovative solutions in the field of treatment of liquid metals, Sidertec made the technological updating of the desulfurization primary system of a major Brazilian integrated facility bringing its system before obsolete to the state of the art of desulfurization and even replacing the desulfurizer $\mathrm{CaC} 2$ the base

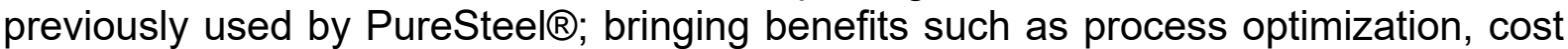
reduction of $61 \%$ and improved operational safety condition by the dangerous material removed from the plant process. This implementation used the Know How Sidertec and was based on the study problem methodology, engineering deepening of possible solutions and implementation of the proposed revamping.
\end{abstract}

Keywords: Dessulphurization; Torpedo car; Technology.

1 Bacharel, Engenheiro Ambiental, Gerente de $P \& D$, Depto de $P \& D$, Sidertec, Sete Lagoas, Minas Gerais, Brasil.

2 Técnico, Técnico em Metalurgia, Diretor, Diretoria Industrial, Sidertec, Sete Lagoas, Minas Gerais, Brasil.

3 Bacharel, Engenheiro Metalúrgico, Gerente Técnico, Departamento Técnico, Sidertec, Sete Lagoas, Minas Gerais, Brasil.

4 Técnico, Técnico em Metalurgia, Técnico de P\&D, Sidertec, Sete Lagoas, Minas Gerais, Brasil

5 Bacharel, Administração de Empresas, Diretor Comercial, Departamento de Vendas e Pós-Vendas, Sidertec, Sete Lagoas, Minas Gerais, Brasil. 


\section{INTRODUÇÃO}

O cenário atual do setor siderúrgico força as usinas e sua cadeia de fornecimento a alcançar índices cada vez mais altos em seus processos, sempre na busca pela melhoria continua da qualidade em seus produtos finais, a maximização da eficiência operacional e de segurança e meio ambiente sendo sobretudo alicerçada pela incansável redução de custos e aumento da competitividade a nível mundial.

Buscando atender com excelência seus clientes através de soluções inovadoras, a Sidertec trabalha para desenvolver as melhores práticas na dessulfuração de ferro gusa em carro torpedo com foco no desenvolvimento de misturas dessulfurantes de alta performance e no desenvolvimento de sistemas de dessulfuração que permitam a seus usuários desfrutar de alta tecnologia e controles avançados que otimizem os processos e seu custo benefício.

Sustentada por esses pilares a Sidertec em conjunto com a equipe técnica do Departamento de Aciaria de uma grande usina integrada situada no Estado de Minas Gerais, desenvolveu estudos e implementou uma rota de dessulfuração em carros torpedo que permitiu a essa unidade flexibilizar seu sistema de dessulfuração.

A unidade em questão possui dois Altos Fornos e utiliza a dessulfuração de ferro gusa através da injeção de dessulfurante a base de $\mathrm{CaC}_{2}$; produto este perigoso e de alto custo, aplicado via unidade cedida em comodato por fornecedor de insumos.

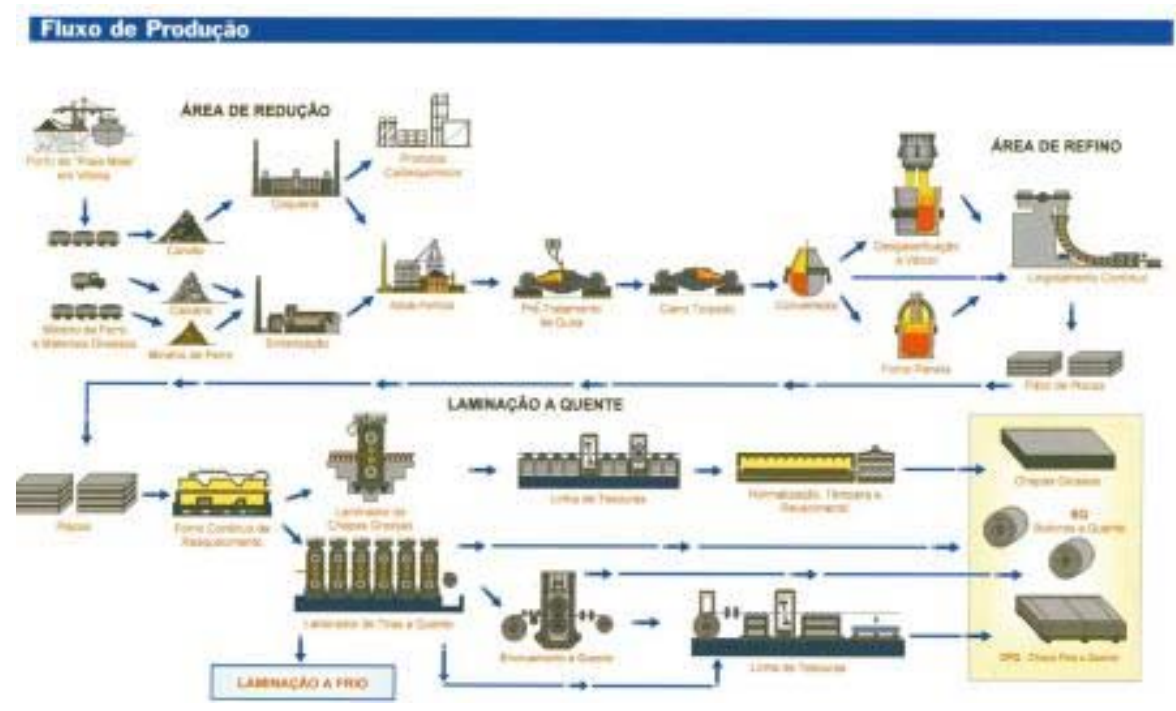

Figura 01 - Fluxo Produtivo Usina Integrada

Neste cenário a Sidertec percebeu a então necessidade do cliente de inicialmente reduzir custos e aumentar competitividade; ter maior poder de barganha através da inserção de outra opção de fornecedor favorecendo assim o trabalho da área de suprimentos e a redução dos riscos operacionais na área de dessulfuração pela substituição do dessulfurante a base da $\mathrm{CaC}_{2}$.

Com esta visão iniciaram se os estudos técnicos voltados para opções que permitissem o atendimento a estes requisitos com o melhor custo benefício ao cliente. Desta forma temos como objetivo deste trabalho apresentar o estudo realizado na unidade siderúrgica, sua implementação e resultados que permitiram ganhos muito interessantes ao cliente. 


\section{MATERIAIS E MÉTODOS}

$\mathrm{Na}$ execução dos trabalhos foi realizada inicialmente uma discussão das opções possíveis para melhoria da eficiência e competitividade da dessulfuração utilizando ferramentas da qualidade, analise estatística dos dados do processo e ainda possibilidades de recursos de engenharia com potencial aplicação.

As ferramentas da qualidade de acordo com Miguel [1] são usadas como apoio ou suporte a decisão na análise de determinado problema.

Ainda de acordo com Miguel [1] as sete ferramentas mais conhecidas da qualidade são: diagrama de causa e efeito, histograma, gráfico de Pareto, diagrama de correlação, gráfico de controle e folha de verificação; sendo escolhida para este estudo a ferramenta diagrama de causa e efeito por ser considerada a mais adequada. Baseado na utilização da ferramenta listamos as atividades a seguir: Determinar o problema a ser estudado, discutir as possíveis causas e listar no diagrama, construir diagrama e agrupar as causas, tomar decisões conforme análise do diagrama e realizar as ações propostas.

Com base nos dados levantados, foi confeccionado o Diagrama de Ishikawa apresentado na figura abaixo que mostram as falhas na competitividade da unidade de dessulfuração primária.

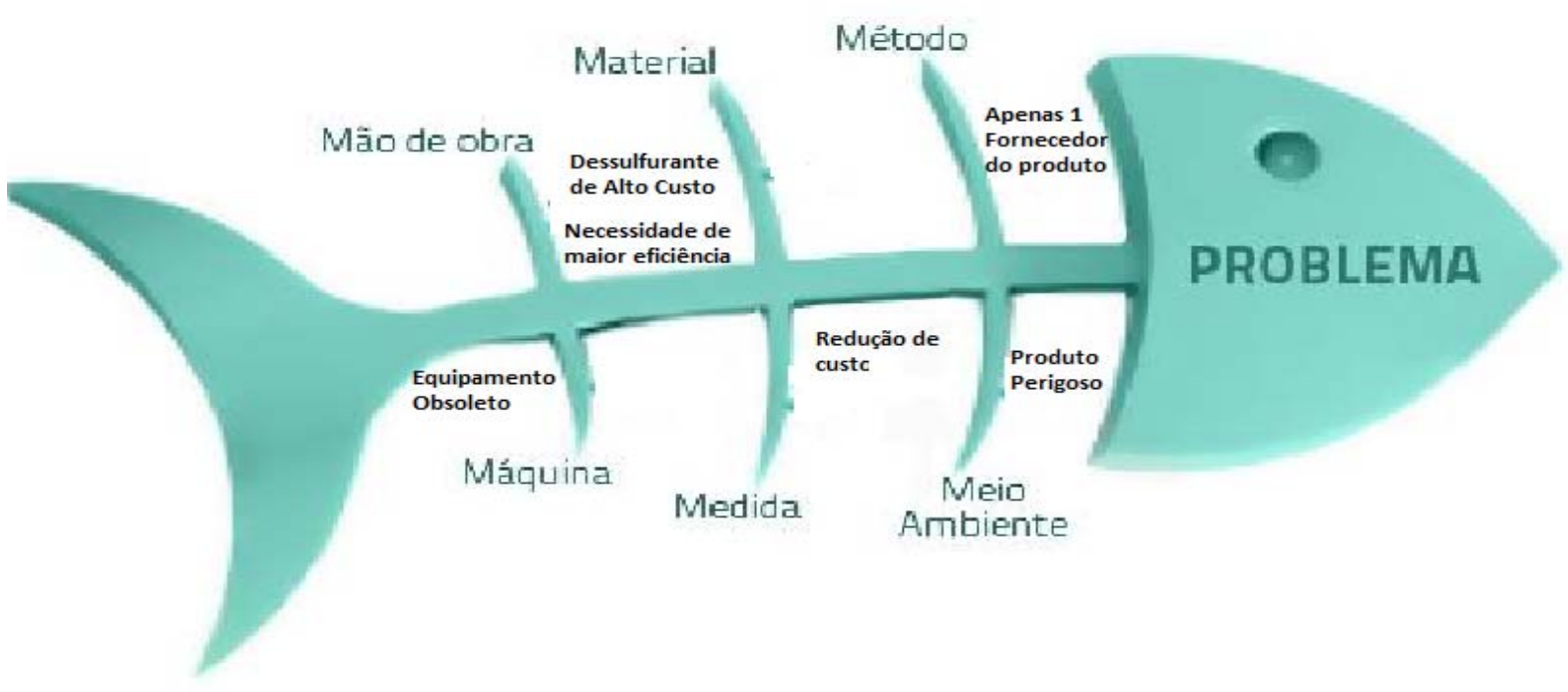

Figura 02 - Diagrama Ishikawa

Assim que levantadas as possíveis causas foram discutidas cada uma conforme o quadro abaixo onde são descritas suas análises e relevância de probabilidade: 
Tabela 1. Analise dos dados (Diagrama Ishikawa)

\begin{tabular}{|c|c|c|}
\hline Causa Provável & Probabilidade & Analise \\
\hline $\begin{array}{l}\text { Dessulfurante de } \\
\text { elevado custo }\end{array}$ & Alta & $\begin{array}{l}\text { Necessidade de } \\
\text { desenvolvimento de } \\
\text { dessulfurante de baixo } \\
\text { custo }\end{array}$ \\
\hline $\begin{array}{l}\text { Necessidade de } \\
\text { maior eficiência }\end{array}$ & Media & $\begin{array}{l}\text { Redução de custo por } \\
\text { menor consumo } \\
\text { especifico do } \\
\text { dessulfurante }\end{array}$ \\
\hline $\begin{array}{l}\text { Apenas } 1 \\
\text { fornecedor do } \\
\text { produto }\end{array}$ & Alta & $\begin{array}{l}\text { Dificuldade de } \\
\text { negociação no setor de } \\
\text { suprimentos }\end{array}$ \\
\hline $\begin{array}{l}\text { Equipamento } \\
\text { Obsoleto }\end{array}$ & Alta & $\begin{array}{l}\text { O equipamento da } \\
\text { usina está obsoleto e } \\
\text { faz com que se deva } \\
\text { utilizar o equipamento } \\
\text { do fornecedor }\end{array}$ \\
\hline Redução de custo & Alta & $\begin{array}{l}\text { A unidade necessita de } \\
\text { competitividade no } \\
\text { tratamento de gusa }\end{array}$ \\
\hline Produto Perigoso & Baixa & $\begin{array}{l}\text { O CaC2 expõe } \\
\text { pessoas e } \\
\text { equipamentos a riscos } \\
\text { de segurança. }\end{array}$ \\
\hline
\end{tabular}

A partir da identificação dos problemas foi definida a solução em conjunto com a unidade siderúrgica sendo:

- Desenvolvimento de produto dessulfurante de baixo custo e alto desempenho

- Atualização tecnológica da unidade de dessulfuração primária

Para atendimento a estes requisitos foram definidas etapas que são:

- Criação do PureSteel 1006: Dessulfurante de alta performance a base de $\mathrm{CaO}$ para aplicação em Dessulfuração em carros torpedo. O material micronizado deste trabalho é fruto de uma patente desenvolvida pela Sidertec com registro no INPI sob o número: BR 102012 013935-9.

- Teste com 30 Toneladas do Puresteel 1006 onde definida a realização de teste para verificação das condições de atendimento; este teste deveria acontecer no sistema considerado obsoleto por suas condições de equipamentos e manter os mesmos parâmetros do produto a base de $\mathrm{CaC}_{2}$.

- Caso o teste com 30 toneladas viesse a ser positivo a Sidertec iria efetuar a Semiautomatização do sistema obsoleto.

- Após realizada a Semi-Automatização a Sidertec procederia um teste com 300 toneladas do PureSteel 1006 a fim de verificar as condições de trabalho em escala industrial.

-Caso o Produto fosse aprovado seria realizada atualização tecnológica completa da dessulfuração primária visando melhores condições operacionais e melhoria nos parâmetros de controle que permitissem maior redução de custo. 
Com os objetivos traçados foi criado o cronograma para realização das atividades, sendo que a Sidertec ainda teve atividades posteriores como a criação de mais duas composições de dessulfurante voltados para maior redução de custo.

Tabela 2. Cronograma de Trabalho

\begin{tabular}{|c|c|c|c|c|c|c|}
\hline \multirow{2}{*}{ Atividades } & \multirow{2}{*}{ Responsável } & \multicolumn{2}{|c|}{2014} & \multicolumn{3}{|c|}{2015} \\
\hline & & 3ㅇ Trimestre & 40 Trimestre & 10 Trimestre & 20 Trimestre & 3ㅇ Trimestre \\
\hline Criação DeS PureSteel 1006 & Sidertec & & & & & \\
\hline Teste 30 ton. Sistema Obsoleto & Sidertec & & & & & \\
\hline Semi Automatização do Sistema & Sidertec & & & & & \\
\hline \multirow{2}{*}{ Teste 300 toneladas PureSteel 1006} & Siderter & & & & & \\
\hline & & & & & & \\
\hline Aprovação PureSteel 1006 & Sidertec & & & & & \\
\hline \multirow{2}{*}{ Revampimg Tecnológico } & & & & & & \\
\hline & Sidertec & & & & & \\
\hline Criação DeS PureSteel 1007 & Sidertec & & & & & \\
\hline & & & & & & \\
\hline Criação PureSteel 1065 & Sidertec & & & & & \\
\hline
\end{tabular}

\section{RESULTADOS E DISCUSSÃO}

Conforme as ações propostas no cronograma, a Sidertec desenvolveu o PureSteel 1006 sob a patente número: BR 102012 013935-9 que deveria ser aplicado num volume de 30 toneladas para verificação da performance. Este produto foi aplicado no sistema obsoleto considerando a dosagem via válvula rotativa e utilizando os mesmos parâmetros do produto a base de CaC2 para manutenção das bases comparativas.

Após realizada a aplicação de 30 toneladas conforme a figura 3 (Gráfico de atendimento ao enxofre) percebeu-se que mesmo com deficiências do sistema de controle o produto atendeu bem aos enxofres requeridos com mesmo consumo especifico do $\mathrm{CaC}_{2}$ tendo apenas como observação a perda média de temperatura que apresentou valor de $31^{\circ} \mathrm{C}$.

$\mathrm{O}$ valor de $31^{\circ} \mathrm{C}$ foi considerado elevado e estabelecida uma meta de $25^{\circ} \mathrm{C}$ para perda de temperatura na dessulfuração em carros torpedo utilizando o PureSteel.

O sistema obsoleto teve bom comportamento durante os testes, porém para garantia do fluxo foi necessária constante intervenção da operação para manter a uniformidade na dosagem; desta forma comprovando as deficiências do sistema. 


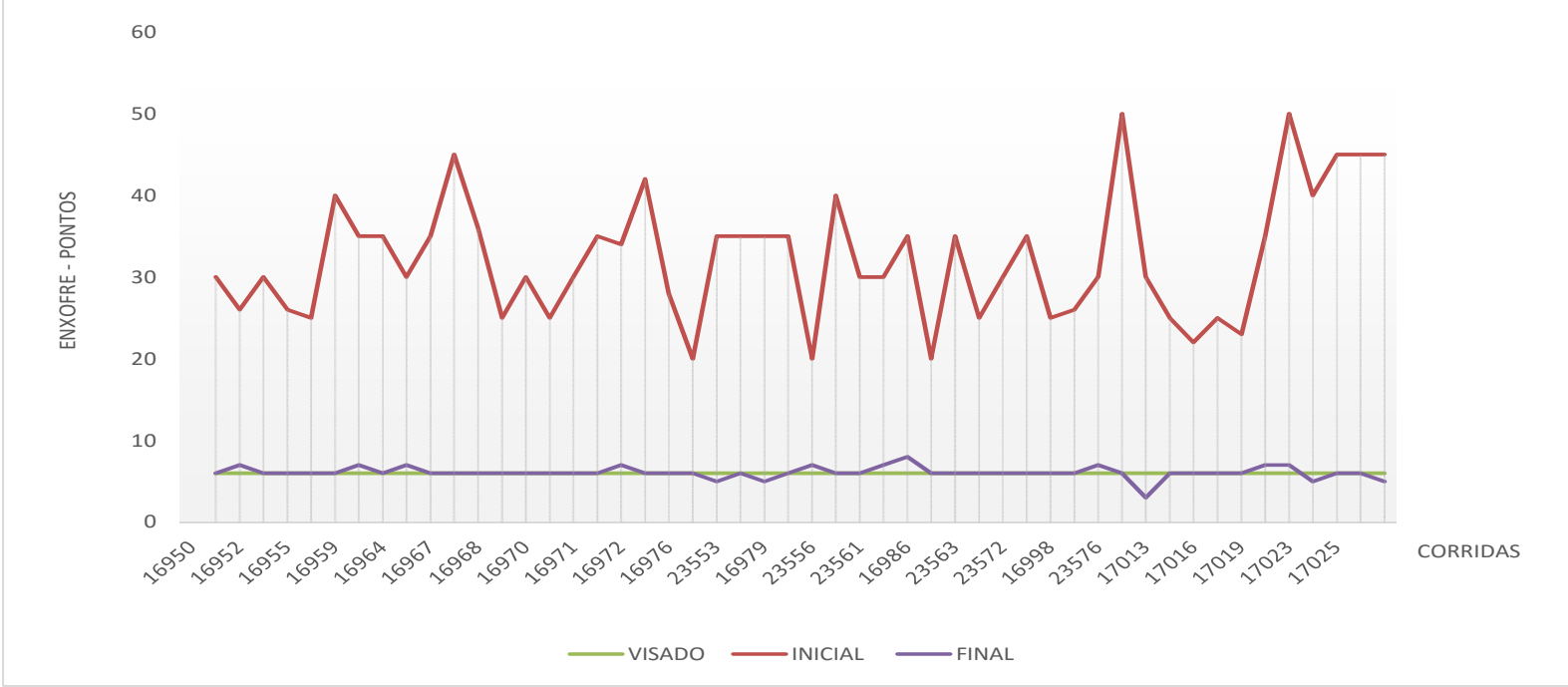

Figura 03 - Gráfico de Atendimento ao Enxofre

Depois de realizada a aplicação e verificados os resultados o teste foi considerado positivo e autorizada a realização de uma aplicação num volume de 300 toneladas para confirmação da performance; onde seriam avaliados os seguintes pontos:

$\checkmark$ Manutenção do Volume de gusa transportado,

$\checkmark$ Perda de Temperatura,

$\checkmark$ Atendimento ao enxofre final

$\checkmark$ Atendimento ao K (Constante Cinética de Processo)

$\checkmark$ Taxa de injeção

$\checkmark$ Consumo especifico

Para o teste de performance com 300 toneladas foi realizada uma semi-automatização do sistema onde foi retirada a válvula rotativa e adaptado um sistema de controle por diferencial de pressão dotado de válvula Shutt off permitindo assim a operação da cabine sem a necessidade da intervenção do operador no decorrer da injeção.

Assim que realizado o teste com acompanhamento técnico da Sidertec foram levantados os dados técnicos onde foram atingidos os índices sobretudo quanto a perda de temperatura onde foi atendida a meta proposta.

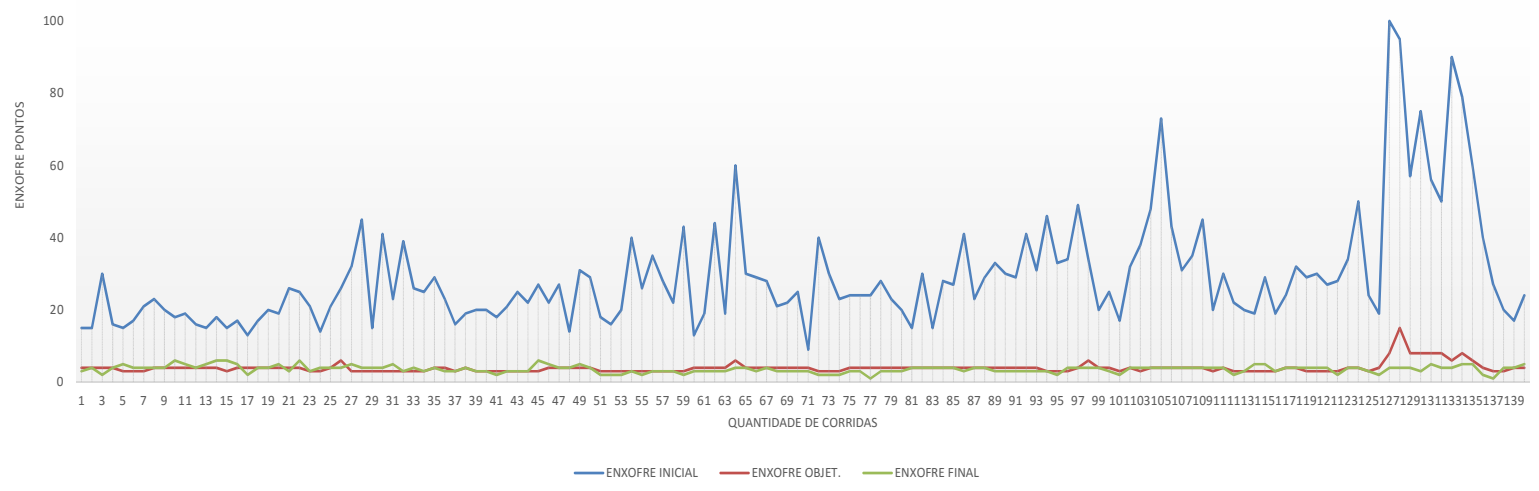

Figura 04 - Gráfico de Atendimento ao Enxofre $2^{\text {a Etapa }}$ 


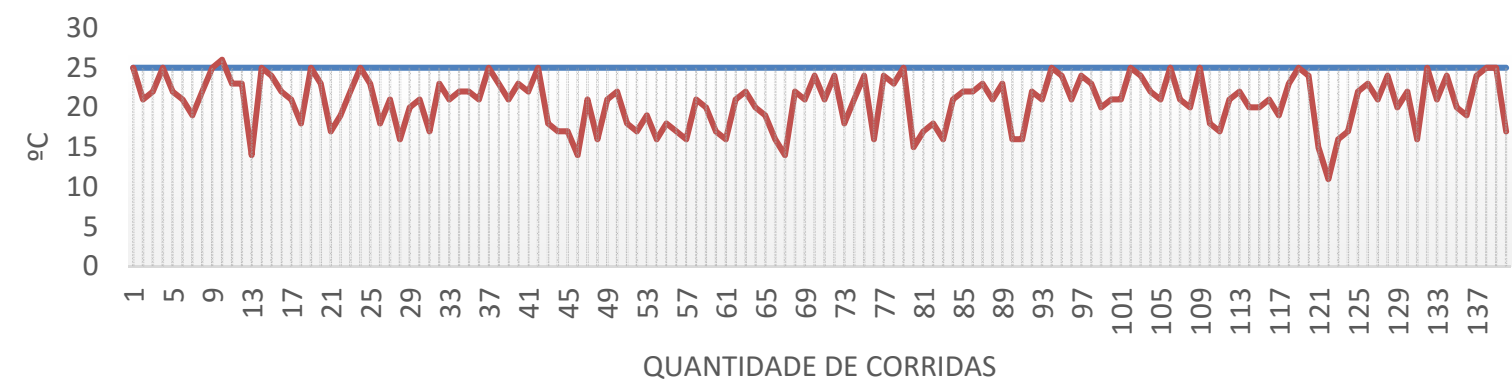

Figura 05 - Gráfico de Perda de Temperatura $2^{\text {a Etapa }}$

Com base nos resultados, na performance de atendimento à aciaria e a redução de custo de aproximadamente 34\%, o produto PureSteel 1006 foi aprovado e aprovada ainda a realização da etapa 3 do projeto que consistia no revamping tecnológico da estação de dessulfuração primaria.

Neste revamping a Sidertec efetuou os seguintes trabalhos:

$\checkmark$ Reforma completa de 2 vasos injetores,

$\checkmark$ Reforma do silo $\mathrm{n}^{\circ} 6 \mathrm{com}$ capacidade de 100 toneladas,

$\checkmark$ Automação do sistema de balanças,

$\checkmark$ Automação do sistema de lanças,

$\checkmark$ Automação do sistema de descarga de carretas

$\checkmark$ Instalação de manifold de gases,

$\checkmark$ Instalação de válvulas reguladoras de pressão e vazão,

$\checkmark$ Instalação de válvula de controle de fluxo (Slidding Disc),

$\checkmark$ Automação completa do sistema com uso de supervisório,

A atualização tecnológica foi realizada de Fevereiro a Julho de 2015 com seu comissionamento em Agosto conforme fotos abaixo.

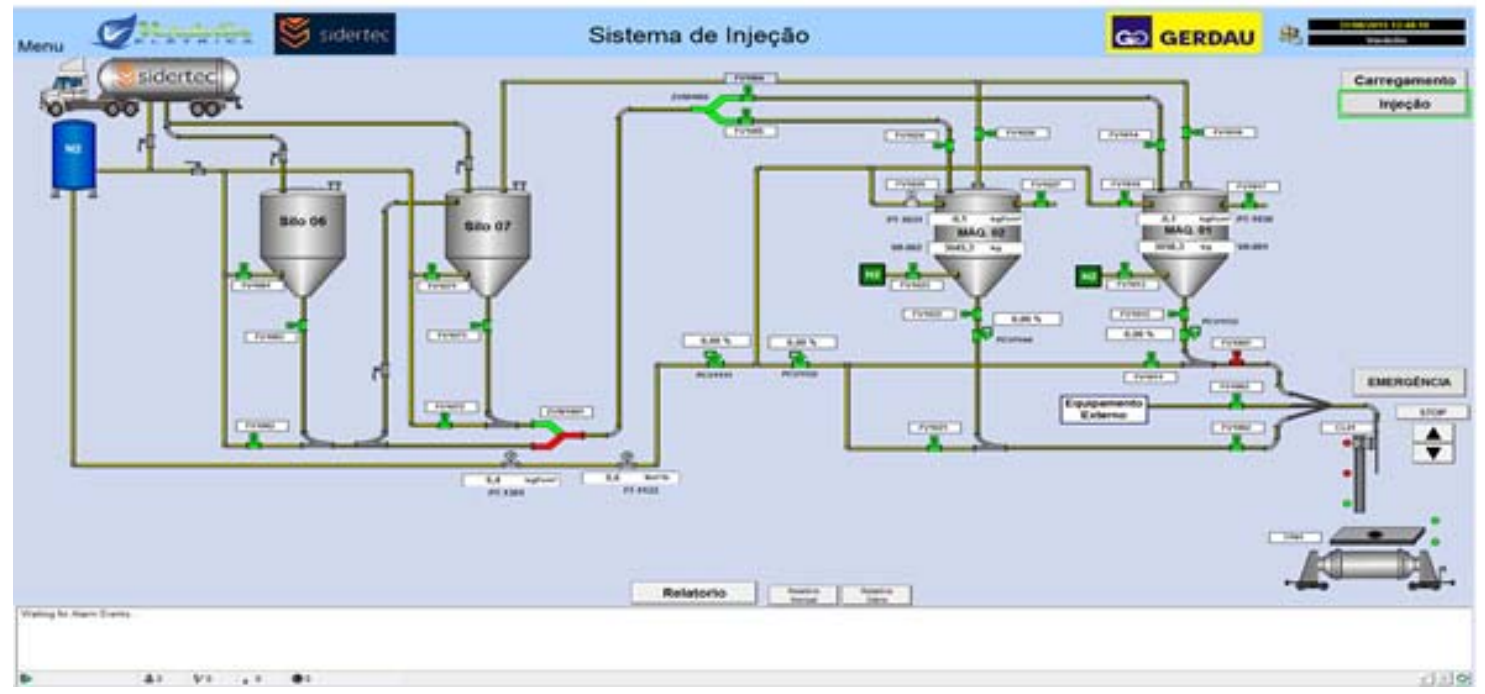

Figura 06 - Tela Principal Supervisório 


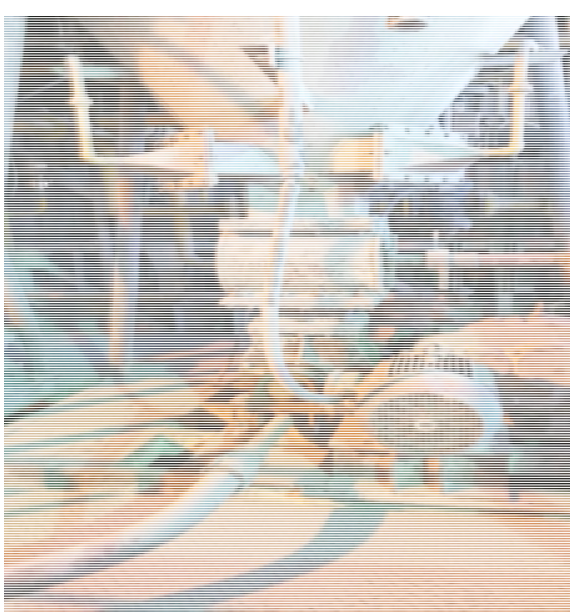

Figura 7 - Dosagem Obsoleta

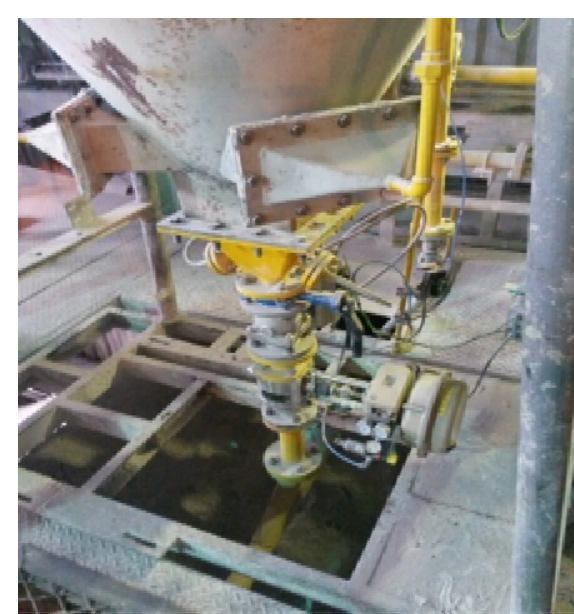

Figura 8 - Dosagem Atual

As melhorias de regularidade de fluxo obtidas com a aplicação da válvula slidding disc permitiram a Sidertec realizar novas composições químicas para atendimento ao processo possibilitando a redução de custo final de $61 \%$ frente ao dessulfurante a base de $\mathrm{CaC}_{2}$ e mantendo as mesmas condições de performance.

\section{CONCLUSÃO}

De acordo com os objetivos propostos para o trabalho listam-se as seguintes considerações:

- A Sidertec proporcionou inovação tecnológica ao seu cliente pelo fornecimento do dessulfurante PureSteel $\AA$ para tratamento de ferro gusa em carro torpedo e pelo fornecimento de Know How no desenvolvimento e instalação de equipamento para dessulfuração;

- Houve uma redução inicial de custo de $34 \%$ no custo de dessulfuração a partir de Outubro de 2014;

- Ocorreu a substituição do dessulfurante a base de $\mathrm{CaC}_{2}$ por PureSteel® dessa forma reduzindo custo e eliminando riscos por se tratar a utilização de produto perigoso;

- Foi aberta a possibilidade de se trabalhar com mais de um fornecedor;

- Comercial e tecnicamente encerrou-se a limitação de uso da dessulfuração primária, pois, a partir do revamping a usina passou a ser detentora do equipamento e não estando mais ligada a equipamento de injeção em comodato;

- Com a Atualização tecnológica foi possível a criação de novas rotas de dessulfuração permitindo a usina ter maior facilidade na programação da aciaria;

- Houve grande percentual de redução de custo com o ajuste da composição do dessulfurante PureSteel $囚$, ganho este permitido graças ao revamping realizado.

Conforme as considerações acima concluem-se que a parceria entre a Sidertec e seu cliente foi vantajosa permitindo ao cliente o aumento da competitividade ao efetuar dessulfurações com custo extremamente reduzido, melhorias na qualidade de seu produto final pelo perfeito atendimento ao enxofre final objetivado, maior segurança na operação e manutenção com a ausência de produto perigoso e a oportunidade de trabalhar com um sistema de dessulfuração no estado da arte.

\section{REFERÊNCIAS}

1 MIGUEL, P.A.C. Qualidade: enfoques e ferramentas, São Paulo, $1^{\text {a }}$ Edição São Paulo, cap.6, pag.225-226, 2006. 